\title{
RECORRÊNCIA EM PACIENTES PORTADORES DE MELANOMA CUTÂNEO SUBMETIDOS A BIÓPSIA DE LINFONODO SENTINELA: SEGUIMENTO MEDIANO DE 37 MESES
}

\author{
PATTERNS OF RECURRENCE FOLLOWING SENTINEL LYMPH NODE BIOPSY FOR \\ CUTANEOUS MELANOMA: OUTCOME AFTER 37 MONTHS OF FOLLOW-UP
}

\author{
Renato Santos de Oliveira Filho, TCBC-SP' ${ }^{1}$ Allisson Monteiro da Silva ${ }^{2}$; \\ Liliana Arcuschin $^{3}$; Jairo Wagner ${ }^{4}$; Lilian Yuri Yamaga ${ }^{5}$
}

\begin{abstract}
RESUMO: Objetivo: A biópsia de linfonodo sentinela (BLS) representa um avanço na cirurgia oncológica para o microestadiamento do melanoma. Apresentamos nossa experiência dando ênfase para a recorrência. Método: A BLS foi realizada em 133 pacientes portadores de melanoma cutâneo localizado envolvendo linfocintilografia, mapeamento linfático e detecção gama intra-operatórios em todos os pacientes. O exame histopatológico foi realizado por HE e imunohistoquímica (IHC). Resultados: Encontrou-se LS em 128 pacientes $(96,2 \%)$. Micrometástase foi diagnosticada em 20 pacientes (15,6\%). Houve nove recorrências, sendo quatro no grupo com LS negativo (108 pacientes). Neste grupo, houve uma recorrência sistêmica e três $(2,8 \%)$ na região linfática de drenagem (falso negativo). No grupo com LS positivo (20 pacientes) ocorreram cinco recorrências. Houve diferença significativa de recorrência entre os grupos, tendo sido menor no grupo LS negativo ( $\mathrm{p}=0,0048$ ). Através de análise de regressão logística univariada a ulceração $(\mathrm{p}=0,029)$ e a positividade do LS $(\mathrm{p}=0,003)$ apresentaram significância estatística como fatores de risco. Porém, apenas a positividade do LS manteve singificância na análise multivariada $(\mathrm{p}=0,024)$. O seguimento mediano foi de 37 meses. Conclusões: Pacientes com LS positivo apresentam recorrência significativamente maior que pacientes com LS negativo. O índice de falso negativo foi de $2,8 \%$ e os pacientes não apresentaram seqüelas o que permite considerar a BLS como procedimento seguro para o microestadiamento do melanoma cutâneo (Rev. Col. Bras. Cir. 2004; 31(5): 279-283) - ISSN 0100-6991.
\end{abstract}

Descritores: Biópsia de Linfonodo Sentinela; Melanoma cutâneo; Linfocintilografia; Mapeamento linfático.

\section{INTRODUÇÃO}

O melanoma cutâneo apresenta alta letalidade e sua incidência vem crescendo em todo o mundo nos últimos 50 anos. Cerca de $90 \%$ dos casos são diagnosticados clinicamente. A percentagem dos pacientes com doença localizada por ocasião do diagnóstico está aumentando ${ }^{1}$.

Na maioria das vezes, os linfonodos são o primeiro sítio de disseminação do melanoma cutâneo ${ }^{2}$. Trata-se de uma disseminação linfonodal randômica de tal forma que nem sempre o linfonodo mais próximo do tumor é o primeiro a receber sua drenagem (linfonodo sentinela), e potencialmente ser comprometido ${ }^{3,4}$.

A biópsia de linfonodo sentinela (LS) define quais pacientes são candidatos apropriados para linfadenectomia completa (aqueles com doença no LS) reservando para seguimento os pacientes com LS sem doença ${ }^{5}$.

Estamos realizando este procedimento desde 1993, inicialmente utilizando o mapeamento linfático com corante vital, de acordo com Morton et al ${ }^{6}$. A partir de 1996, acrescentamos o uso da detecção gama intra-operatória ${ }^{7}$. Realizou-se um seguimento clínico rigoroso destes pacientes, permitindo avaliar a recorrência entre os mesmos, objetivo principal deste trabalho.

\section{MÉTODO}

Cento e trinta e três pacientes portadores de melanoma cutâneo, 63 homens e 70 mulheres, com idade mediana de 49 anos (18 - 86 anos) foram submetidos à biópsia de linfonodo sentinela de acordo com protocolo estabelecido, entre agosto de 1996 e maio de 2003. De acordo com este protocolo, a biópsia de linfonodo sentinela foi realizada para pacientes portadores de melanoma localizado, com espessura de Breslow maior ou igual a 1,0 $\mathrm{mm}$ ou com espessura de Breslow menor que $1,0 \mathrm{~mm}$, porém com nível de Clark IV ou V ou ulceração na lesão primária ${ }^{8,9}$. As características gerais destes pacientes podem ser observadas na Tabela 1.

A linfocintilografia pré-operatória foi realizada em todos os pacientes. Para a biópsia de LS foram utilizados o mapeamento linfático com corante azul patente $\mathrm{V}$ e a detecção gama intra-operatória (Neoprobe 1500). Os linfonodos foram examinados para pesquisa de micrometástase pelo método convencional (HE) e imunohistoquímico (IHC). Sugeriu-se, aos

1. Cirurgião Oncológico, Professor Adjunto Visitante e Professor Orientador de Teses do Programa de Pós-Graduação em Cirurgia Plástica Reparadora da EPM/UNIFESP.

2. Cirurgiã de Cabeça e Pescoço, Mestre em Medicina pela EPM/UNIFESP.

3. Diretora Clínica do Centro Avançado de Prevenção e Tratamento de Câncer - São Paulo.

4. Coordenador do Serviço de Medicina Nuclear do Hospital Israelita Albert Einstein.

5. Médica Nuclear do Serviço de Medicina Nuclear do Hospital Israelita Albert Einstein, Doutora em Medicina pela Universidade de São Paulo. Recebido em 12/08/2003

Trabalho realizado no Centro Avançado de Prevenção e Tratamento de Câncer e no Hospital Israelita Albert Einstein, São Paulo, Brasil. 
Tabela 1 - Características clínico-patológicas dos 133 pacientes.

\begin{tabular}{ll}
\hline Variável & n \\
\hline Idade mediana & $49(18-86)$ anos \\
Sexo (M/F) & $63 / 70$ \\
Localização da lesão primária & \\
Cabeça e Pescoço & 18 \\
Tronco & 57 \\
Membro Superior & 24 \\
Membro Inferior & 34 \\
Tipo Histopatológico & \\
Disseminativo Superficial & 79 \\
Nodular & 41 \\
Acral & 7 \\
Lentigo Maligno Melanoma & 2 \\
Outros & 4 \\
Breslow & \\
<1 mm & 35 \\
1-4 mm & 81 \\
>4mm & 9 \\
Prejudicado & 8 \\
Clark & \\
<=III & 72 \\
>=IV & 61 \\
Ulceração & \\
Presente & 108 \\
Ausente & 5 \\
Prejudicado & \\
LS & \\
Positivo & \\
Negativo & \\
Não avaliado & \\
\hline
\end{tabular}

pacientes com LS comprometido, a realização de linfadenectomia completa e aos demais apenas seguimento clínico. Todos os pacientes foram operados e seguidos pela mesma equipe médica. Para a realização da linfocintilografia pré-operatória, uma dose de 250 miliCuries do radiofármaco foi administrada intradermicamente em quatro regiões, seguindo-se a orientação dos quatro pontos cardeais, bem próximo da lesão ou da cicatriz, num volume total de $1 \mathrm{ml}$. As imagens de sua biodistribuição foram tomadas em câmera de cintilação modelo Starcam 4000600 X/RT, inicialmente a cada 15 segundos até a identificação do linfonodo sentinela. Após esta fase dinâmica, novas imagens foram adquiridas a cada 15 minutos, até uma hora. Uma imagem tardia de duas horas também foi realizada, tomando-se o cuidado de varrer todas as bases linfáticas prováveis de receber migração do radiofármaco. $\mathrm{O}$ radioisótopo utilizado foi o $\mathrm{Tc}^{99}$ ligado ao dextran $500 \mathrm{ou}$ ao fitato.

Com o paciente posicionado na mesa cirúrgica, 1 a 2 $\mathrm{ml}$ de azul patente $\mathrm{V}$ (Laboratório Guerbet) foi injetado intradermicamente ao redor da cicatriz ou melanoma, para a realização do mapeamento linfático. Cerca de 10 a 15 minutos após, uma incisão de aproximadamente $2 \mathrm{~cm}$ foi feita sobre o local marcado pela linfocintilografia pré-operatória, corrigido pela detecção gama intra-operatória. Desta forma, a cicatriz poderia ser incorporada numa posterior linfadenectomia completa, caso a mesma viesse a ser necessária. Através de dissecção cuidadosa dos retalhos cutâneos e do tecido subcutâneo, os vasos linfáticos aferentes foram identificados, dissecados e seguidos até se encontrar o LS o qual se apresentava com coloração azul. Todos os linfonodos corados foram retirados.

Na maioria das vezes a detecção gama intra-operatória foi realizada aproveitando-se a mesma injeção de radiofármaco feita para a linfocintilografia pré-operatória. Através das leituras das escalas numéricas e orientação sonora pelo aparelho de DGI (Neoprobe 1500), definiu-se o local de incisão da pele, e orientou-se o encontro e exérese do LS. Os LS retirados foram aferidos pelo detector portátil de radiação gama, o mesmo se fazendo para o leito operatório e local fora de trajeto de grandes vasos e longe da região linfática abordada e do sítio primário (leitura de fundo). Quando a captação do leito operatório era maior que $10 \%$ da leitura ex-vivo do LS, continuou-se a procura e retirada de outro LS.

A pesquisa de micrometástase foi feita através de exame histopatológico convencional (HE) e estudo imunohistoquímico (HMB 45, proteína S-100, MART-1).

Análise estatística foi realizada utilizando-se o teste exato de Fisher e regressão logística com análise uni e multivariada.

\section{RESULTADOS}

Todos os pacientes fizeram linfocintilografias préoperatórias. Das 133 linfocintilografias realizadas, em um paciente não se observou migração do radiotraçador, em um paciente houve drenagem para três regiões linfáticas, em 13 houve drenagem para duas e nos demais a drenagem ocorreu para apenas uma região linfática. Em um paciente houve migração para mediastino e em outro para o retroperitônio, que não foram explorados. As regiões linfáticas exploradas foram axilar (81), inguinal (39), cervical (18), parotídea (3) e subescapular (6).

Utilizamos ambos o corante vital e a detecção gama intra-operatória e encontramos o LS em 128 dos 133 pacientes $(96,2 \%)$. Foram encontrados 199 LS nas 147 regiões linfáticas exploradas (1,4 LS / região linfática explorada), sendo que em dois pacientes explorados não se encontrou o LS. Micrometástase foi diagnosticada em 20 pacientes $(15,3 \%)$. Destes, 19 foram submetidos à linfadenectomia completa sendo que em quatro (21\%) encontrou-se mais um linfonodo com micrometástase. O outro paciente recusou a linfadenectomia e foi submetido à radioterapia na área de drenagem (cervical). O diagnóstico de micrometástase no LS foi dado pela HE e IHC em 17 pacientes, e nos outros três, apenas pela IHC (HMB 45 e proteína $S$ 100). A HE foi feita em todos os casos e a imunohistoquímica foi realizada em 93 pacientes. Na Tabela 2 podemos observar a recorrência em função da espessura de Breslow, nível de Clark, ulceração na lesão primária e comprometimento do LS. 
Ao todo, nove pacientes apresentaram recorrência. Entre os 108 com LS negativo houve quatro recorrências, três $(2,8 \%)$ na região linfática de drenagem (falso negativo) e uma sistêmica. Entre os 20 pacientes com LS positivo ocorreram cinco recorrências, todas sistêmicas sendo que um deles também apresentou metástase em trânsito. Quatro dos pacientes com recorrência sistêmica evoluiram para o óbito, os outros dois estão vivos com doença e outro paciente que não tinha recorrência de melanoma faleceu por câncer de próstata.

Em 15 pacientes foram observados seromas, hematomas, ou hipoestesia, que foram resolvidos sem deixar seqüelas. Em três pacientes houve tatuagem permanente (mais que seis meses) pelo azul patente e em dois pacientes

Tabela 2 - Recorrência e fatores de risco.

\begin{tabular}{lccc}
\hline Variável & $\begin{array}{c}\text { Com } \\
\text { recorrência }\end{array}$ & $\begin{array}{c}\text { Sem } \\
\text { recorrência }\end{array}$ & p \\
\hline $\begin{array}{l}\text { Breslow } \\
\quad<1,0 \mathrm{~mm}\end{array}$ & 1 & 34 & $\mathrm{p}=0,27$ \\
$\quad \begin{array}{l}\text { 1,0 mm } \\
\text { Ulceração }\end{array}$ & 8 & 82 & \\
$\quad$ Sim & 4 & 17 & $\mathrm{p}=0,045^{*}$ \\
$\quad$ Não & 5 & 97 & \\
LS & & & \\
$\quad$ Positivo & 5 & 15 & $\mathrm{p}=0,0048^{*}$ \\
$\quad$ Negativo & 4 & 104 & \\
\hline Teste de Fisher . Significância estatística $(p<0,05)$. &
\end{tabular}

Tabela 3 - Regressão logística univariada e multivariada (Recorrência vs Ulceração ou Breslow ou Clark ou LS).

\section{UNIVARIADA}

\begin{tabular}{|c|c|c|}
\hline Variável & $\begin{array}{l}\text { Odds ratio } \\
(95 \% \text { CI })\end{array}$ & $\mathbf{p}$ \\
\hline $\begin{array}{l}\text { Breslow } \\
\qquad<1,0 \text { vs } \geq 1,0\end{array}$ & $\begin{array}{c}2,77 \\
(0,33-23,38)\end{array}$ & 0,350 \\
\hline $\begin{array}{l}\text { Clark } \\
\qquad \geq 4 \text { vs } \leq 3\end{array}$ & $\begin{array}{c}1,48 \\
(0,35-6,20)\end{array}$ & 0,595 \\
\hline $\begin{array}{l}\text { Ulceração } \\
\text { Sim vs Não }\end{array}$ & $\begin{array}{c}4,85 \\
(1,17-20,01)\end{array}$ & $0,029 *$ \\
\hline $\begin{array}{l}\text { LS } \\
\qquad \text { Positivo vs Negativo }\end{array}$ & $\begin{array}{c}8,67 \\
(2,09-35,92)\end{array}$ & $0,003 *$ \\
\hline
\end{tabular}

\section{MULTIVARIADA}

\begin{tabular}{|c|c|c|}
\hline Variável & $\begin{array}{l}\text { Odds ratio } \\
(95 \% \mathrm{CI})\end{array}$ & $\mathbf{p}$ \\
\hline $\begin{array}{l}\text { Ulceração } \\
\text { Sim vs Não }\end{array}$ & $\begin{array}{c}2,09 \\
(0,398-10,96)\end{array}$ & 0,384 \\
\hline $\begin{array}{l}\text { LS } \\
\qquad \text { Positivo vs Negativo }\end{array}$ & $\begin{array}{c}6,40 \\
(1,27-32,19)\end{array}$ & $0,024 *$ \\
\hline
\end{tabular}

* significância estatística $(p<0,05)$ houve uma reação inflamatória no local de injeção do radiofármaco. O seguimento mediano foi de 37 meses (três a 77 meses).

\section{DISCUSSÃO}

O manejo dos pacientes portadores de melanoma cutâneo localizado, porém com risco de desenvolver metástase regional tem sido tema de controvérsia durante décadas. Sob o ponto de vista da prática clínica atual, o procedimento de biópsia de LS põe fím à grande controvérsia entre realizar linfadenectomia eletiva ou apenas observar estes pacientes. Com este procedimento, somente os pacientes com LS comprometido são submetidos à linfadenectomia completa ${ }^{3}$.

Embora a utilização do corante vital e da detecção gama intra-operatória se complementem, é preciso destacar que o aprendizado da biópsia de LS com o corante é mais lento do que com a detecção gama intra-operatória ${ }^{10,11}$. Por outro lado, a utilização apenas do corante vital é muito mais econômica e pode ser suficiente em muitas situações, como quando a linfocintilografia pré-operatória mostra drenagem apenas para a região inguino-crural superficial ${ }^{12}$.

Nossa taxa de encontro do LS foi de $96,2 \%$. O LS se mostrou negativo em 108 pacientes e nestes houve quatro recorrências, sendo três $(2,8 \%)$ na região linfática de drenagem (falso negativo) e uma sistêmica, reforçando o conceito de que inicialmente o melanoma se dissemina para o linfonodo sentinela, daí para o restante da região linfática e posteriormente para outros órgãos ${ }^{13}$.

Entre os pacientes com LS negativo a recorrência foi menor do que entre os com LS positivo ( $p=0,0048$; teste de Fisher). Também foi significativa a maior recorrência entre os pacientes com ulceração na lesão primária $(\mathrm{p}=0,045$; teste de Fisher). Na análise de regressão logística univariada, a ulceração $(\mathrm{p}=0,029)$ e a positividade do LS $(\mathrm{p}=0,003)$ tiveram significância estatística como fator de risco, porém apenas o LS manteve significância na análise multivariada ( $\mathrm{p}=0,024)$. Dos 20 pacientes com LS positivo um apresentava Breslow menor que $1,0 \mathrm{~mm}$ sendo que a espessura de Breslow (menor que 1,0 mm versus maior ou igual a 1,0 mm) não apresentou significância estatística em relação à recorrência do melanoma (Tabela 3). Outros achados na lesão primária, além da espessura de Breslow, nível de Clark e ulceração, tais como regressão, alto índice mitótico (acima de seis mitoses $/ \mathrm{mm}^{2}$ ), fase de crescimento vertical e invasão linfática estão sendo valorizados como fatores de risco ${ }^{9,14-16}$.

Métodos baseados em reação de polimerase em cadeia por transcriptase reversa (RT-PCR) têm sido usados para o diagnóstico de micrometástases objetivando diminuir os resultados falso-negativos ${ }^{17,18}$. A relevância das micrometástases detectadas por RT-PCR na recorrência do melanoma não está ainda bem estabelecida. Uma revisão sistemática da literatura médica com metanálise mostrou que o diagnóstico de micrometástase por RT-PCR exclusivo tem valor de pior prognóstico para estes pacientes ${ }^{19}$. O inconveniente desta técnica é que ainda apresenta altos índices de fal- 
so-positivos o que pode ser diminuído pela utilização de múltiplos marcadores ${ }^{20,21}$.

Entre os 19 pacientes submetidos à linfadenectomia completa por apresentarem LS positivo, quatro (21\%) tiveram outro linfonodo comprometido, sendo que todos apresentavam espessura de Breslow maior que 1,5 mm. Ainda não se têm fatores identificados, no LS positivo, capazes de prever com segurança a ocorrência de outro linfonodo positivo no produto da linfadenectomia completa. Portanto, recomendase a linfadenectomia completa para todos os pacientes com LS positivo.

Até o presente momento, o benefício de sobrevida da utilização da técnica de biópsia de LS não foi confirmado através de ensaios clínicos, como o Multicenter Selective Lymphadenectomy Trial, nos EUA. A conclusão deste estudo não é esperada antes de 2005 e nele os pacientes eleitos são randomizados em dois grupos: um recebe apenas o tratamento de lesão primária e o outro acrescenta a biópsia de LS. Outro estudo em andamento muito importante é o Sunbelt Melanoma Trial, também nos EUA, que procura avaliar o significado clínico da doença linfonodal microscópica, determinada pela histopatologia convencional, imunohistoquímica e análise por RT-PCR de qua- tro marcadores moleculares (MAGES III, MART-1, GP 100 e Tirosinase) e o efeito do interferon na micrometástase. Estudo semelhante está sendo conduzido pela European Organization for Research and Treatment of Câncer. Embora tenha seguido um protocolo, nosso trabalho não teve randomização não permitindo avaliar se houve ou não benefício na sobrevida dos pacientes submetidos a biópsia de linfonodo sentinela.

A recorrência, na nossa casuística, foi significativamente maior nos pacientes com LS positivo ( $\mathrm{p}=0,0048)$. Nesse sentido, a linfadenectomia complementar parece controlar a região linfática. $\mathrm{O}$ índice de falso negativo foi de $2,8 \%$, sendo que este valor ainda apresenta grande variação na literatura ${ }^{22}$. Os pacientes não apresentaram seqüelas o que permite considerar a BLS como procedimento seguro para o microestadiamento do melanoma cutâneo.

Imunohistoquímica e RT-PCR com a utilização de diversos marcadores tumorais deverão ser incorporados à rotina do exame do LS, contribuindo para diminuir os casos de falso negativo, aumentando a acurácia da biópsia de linfonodo sentinela no microestadiamento do melanoma. O desenvolvimento da robótica poderá facilitar o acesso a regiões como mediastino e retroperitônio.

\begin{abstract}
Background: Sentinel node biopsy (SNB) has been considered as an advance in surgical oncology for microstading melanoma. We report our experience with this procedure focusing on recurrence. Methods: SNB was performed in 133 cutaneous melanoma patients submitted to preoperative lymphoscintigraphy, lymphatic mapping and gamma probe detection. Histologycal samples were analysed by HE and immunohistochemistry (IHC). Results: The sentinel node was detected in 126 patients (96.2\%). Micrometastasis were diagnosed in 20 patients (15.6\%). There were nine recurrence, four in negative sentinel node group (108 patients). In this group, there was one systemic recurrence and three (2.8\%) on lymphatic drainage region (false negative). In positive sentinel node group (20 patients) there were five recurrences. The recurrence was lower among negative sentinel node patients $(p=0.0048)$. Ulceration $(p=0.029)$ and positivity of the sentinel node $(p=0.003)$ were considered significant risk factor by logistic analisys. Only sentinel node positivity maintained significance on multivariate analysis $(p=0.024)$. The median follow up was 37 months. Conclusions: Patients with positive sentinel node presented more recurrence than negative sentinel node patients. The false negative rate was $2.8 \%$ and patients didn't have long-term sequelae, allowing us to consider SNB as a secure and accurate procedure for melanoma staging.
\end{abstract}

Key words: Sentinel node biopsy; Cutaneous melanoma; Lymphoscintigraphy; Lymphatic mapping.

\section{REFERÊNCIAS}

1. Brozena SJ, Fenske NH, Perez IR - Epidemiology of malignant melanoma, worldwide incidence and etiologic factors. Semin Surg Oncol, 1993, 9(3):165-173.

2. Reintgen D, Cruse CW, Wells K, et al. - The orderly progression of melanoma nodal metastases. Ann Surg, 1994, 220(6):759-767.

3. Oliveira-Filho RS, Paiva GR, Wagner J - "Biópsia de linfonodo sentinela no melanoma cutâneo". In Oliveira-Filho RS (ed) Melanoma cutâneo localizado e linfonodo sentinela. 1 ${ }^{a}$ Edição. São Paulo, Lemar Editora, 2003, pp.113-124.

4. Norman J, Cruse CW, Epinosa C, et al. - Redefinition of cutaneous lymphatic drainage with the use of lymphoscintigraphy for malignant melanoma. Am J Surg, 1991, 162(5):432-437.

5. Morton DL, Thompson JF, Essner R, et al. - Validation of the accuracy of intraoperative lymphatic mapping and sentinel lymphadenectomy for early-stage melanoma: a multicenter trial. Multicenter Selective Lymphadenectomy Trial Group. Ann Surg, 1999, 230(4):453-465.
6. Morton DL, Wen DR, Wong JH, et al. - Technical details of intraoperative lymphatic mapping for early-stage melanoma. Arch Surg, 1992,127(4):392-399

7. Oliveira-Filho RS, Almeida FA, Santos IA, et al. - Linfadenectomia seletiva com biópsia de linfonodo sentinela no melanoma cutâneo. Experiência brasileira - Projeto FAPESP 97/2516-0. An Bras Dermatol, 2000, 75(5):573-580.

8. Bedrosian I, Faries MB, Guerry D, et al. - Incidence of sentinel node metastasis in patients with thin primary $(<=1 \mathrm{~mm})$ with vertical growth phase. Ann Surg Oncol, 2000, 7(4):262-267.

9. Oliveira Filho RS, Ferreira LM, Biasi LJ, et al. - Vertical growth phase and positive sentinel node in thin melanoma. Braz J Med Biol Res, 2003, 36(3):347-350.

10. Kapteijn BA, Nieweg OE, Liem I, et al. - Localizing the sentinel node in cutaneous melanoma: gamma probe detection versus blue dye. Ann Surg Oncol, 1997, 4(2):156-160.

11. Pijpers R, Borgstein PJ, Teule GJ, et al. - Vital dye and radiolabelled colloides-complement or alternative? Recent Results Cancer Res, 2000,157:130-137. 
12. Oliveira-Filho RS, Santos ID, Ferreira LM, et al. - Is intraoperative gamma probe detection really necessary for inguinal sentinel lymph node biopsy? São Paulo Med J, 2000, 118(6):165168.

13. Chao C, Wong SL, Ross MI, et al. - Patterns of early recurrence after sentinel lymph node biopsy for melanoma. Am J Surg, 2002,184(6):520-525.

14. Slingluff CL, Vollmer RT, Reintgen DS, et al. - Lethal "thin" malignant melanoma. Identifying patients risk. Ann Surg, 1988, 208(2):150-161.

15. Thompson JF, Shaw HM - Sentinel node metastasis from thin melanomas with vertical growth phase. Ann Surg Oncol, 2000, 7(4):251-252.

16. Fearfield LA, Rowe A, Francis N, et al. - Clinico-pathological features of relapsing very thin melanoma. Clin Exp Dermatol, 2001, 26(8):686-695.

17. Blaheta HJ, Schittek B, Breuninger H, et al. - Detection of melanoma micrometastase in sentinel nodes by reverse transcription-polymerase chain reaction correlates with tumor thickness and is predictive of micrometastatic disease in the lymph node basin. Am J Surg Pathol, 1999, 23(7):822828.

18. Cochran AJ, Huang RR, Guo J, et al. - Current practice and future directions in pathology and laboratory evaluation of the sentinel node. Ann Surg Oncol, 2001, 8(Suppl 9):13S-17S.
19. da Silva AM, Oliveira-Filho RS, Ferreira LM, et al. - Relevance of micrometastases detected by reverse transcripatase polymerase chain reaction for melanoma recurrence: systematic review and meta-analysis. São Paulo Med J, 2003, 121(1):2427.

20. Davids V, Kidson SH, Hanekom GS - Melanoma patient staging: histopathological versus molecular evaluation of the sentinel node. Melanoma Res, 2003,13(3):313-324.

21. Goydos JS, Ravikumar TS, Germino FJ, et al. - Minimally invasive staging of patients with melanoma: sentinel lymphadenectomy and detection of the melanoma-specific proteins MART-1 and tyrosinase by reverse transcriptase polymerase chain reaction. $\mathrm{J}$ Am Coll Surg, 1998,187(2):182-190.

22. Estourgie SH, Nieweg OE, Valdes Olmos RA, et al. - Review and evaluation of sentinel node procedures in 250 melanoma patients with a median follow-up of 6 years. Ann Surg Oncol, 2003,10(6):681-688.

Endereço para correspondência:

Renato Santos de Oliveira Filho

Av.Rebouças, 3084, Cj.14

05402-600 Pinheiros - São Paulo - SP

Email: renato.dcir@unifesp.epm.br

Tel: (11) 3031-7681; Fax: (11) 3032-0763 